\title{
On the Slow Viscous Flow through a Swarm of Solid Spherical Particles Covered by Porous Shells
}

\author{
Pramod Kumar Yadav
}

Department of Mathematics, National Institute of Technology Patna, Patna, 800005, Bihar, India

\begin{abstract}
This paper concerns the slow viscous flow through a swarm of concentric clusters of porous spherical particles. An aggregate of clusters of porous spherical particles is considered as a hydro-dynamically equivalent to a porous spherical shell enclosing a solid spherical core. The Brinkman equation inside and the Stokes equation outside the porous spherical shell in their stream function formulations are used. As boundary conditions, continuity of velocity, continuity of normal stress and stress-jump condition at the porous and fluid interface, the continuity of velocity components on the solid spherical core are employed. On the hypothetical surface, uniform velocity and Happel boundary conditions are used. The drag force experienced by each porous spherical shell in a cell is evaluated. As a particular case, the drag force experienced by a porous sphere in a cell with jump is also investigated. The earlier results reported for the drag force by Davis and Stone[5] for the drag force experienced by a porous sphere in a cell without jump, Happel[2] for a solid sphere in a cell and Qin and Kaloni[4] for a porous sphere in an unbounded medium have been then deduced. Representative results are presented in graphical form and discussed.
\end{abstract}

Keywords Spherical Porous Shell, Cell Model, Permeability, Brinkman Equation, Drag Force

\section{Introduction}

The study of viscous flow through a porous medium has gained importance in recent years because of its natural occurrence and of its importance in bio-mechanics, physical sciences and chemical engineering etc. The flow of fluids through a swarm of porous particles has many industrial and engineering applications, such as, in flow through porous beds, in petroleum reservoir rocks, in flow sedimentation, etc. Several researchers have considered the flow of viscous fluid past and through solids or porous bodies with different models. For effective use of a porous medium in the above areas, the structure of porous layer should be viewed from all angles. There are many physical situations arises in which the porous particles moving in the viscous fluid. The flow in most of the above process is creeping because the Reynolds number is smaller than unity. The two terms that play an important role for analytical treatment of the problems related to flow through porous media, are porosity and permeability. The porosity The porosity represents the ratio of volume of openings (voids or pores) to the volume of the material. It seems that more the number of pores, easier will be the flow through the medium, which is not correct and this can be explained by permeability. Permeability is a measure

* Corresponding author:

pramod547@gmail.com (Pramod Kumar Yadav)

Published online at http://journal.sapub.org/am

Copyright (C) 2011 Scientific \& Academic Publishing. All Rights Reserved of interconnectivity of voids (pores) in the medium. Hence, its number of voids along with their interconnectivity both that determine the ease with which fluid will flow through the medium. For the medium of high porosity, the sum suggested by Brinkman[1] is more suitable for describing the flow through the porous medium. Brinkman[1] evaluated the viscous force exerted by a flowing fluid on a dense swarm of particles by introducing modified Darcy's equation for porous medium, which is commonly known as Brinkman equation.

In the analysis of flow through swarm of particles, we get cumbersome calculations, if we consider the solution of the flow field over the entire swarm by taking exact positions of the particles. In order to avoid the above complication, it is sufficient to obtain the analytical expression by considering the effects of the neighboring particles on the flow field around a single particle of the swarm, which can be used to develop relatively simple and reliable models for heat and mass transfer. This has lead to the development of particle-in-cell models.

Uchida[2] proposed a cell model for a sedimenting swarm of particles, considering spherical particle surrounded by a fluid envelope with cubic outer boundary. This was accurately solved by Brenner[3]. Happel[4, 5] proposed cell models in which both particle and outer envelope are spherical. Happel solved the problem when the inner sphere is solid with respective boundary conditions on the cell surface. The Happel model assumes uniform velocity condition and no tangential stress at the cell surface. The merit of this 
formulation is that, it leads to an axially symmetric flow that has a simple analytical solution in closed form, and thus can be used for heat and mass transfer calculations. Analytical solutions of particle-in-cell models discussed above are always practically useful to many industrial problems, but the solutions of creeping flow for the above models have not been found in case of complex geometry. However, for geometrically simpler cases like sphere, the analytical solutions were investigated by various authors along with Happel. The problem of the slow viscous flow through an aggregate of concentric clusters of porous cylindrical particles with Happel boundary condition was discussed Deo et al[6]. The drag force experienced by each porous circular cylindrical particle in a cell was evaluated them by using cell model technique

Jones[7] had investigated the solution of the problem of Stokes flow past a porous spherical shell. He used Darcy's law for the porous region and evaluated the drag force experienced by a shell. A Cartesian-tensor solution of the Brinkman equation is investigated by Qin and Kaloni[8] and they also evaluated the drag force on a porous sphere in an unbounded medium. Flow through beds of porous particles was studied by Davis and Stone[9]. They present two approximate models for describing the flow field within a porous particle contained in a fixed or fluidized bed.

The first is a swarm model where a spherical porous particle of a specified permeability is contained in a matrix of a different permeability that is equated to the overall bed permeability. The second is a cell model in which the spherical porous particle is placed in a spherical envelope of fluid whose diameter is chosen so that the overall bed porosity is conserved The focus of their work is to derive an expressions for the velocity and pressure profiles inside and outside of the porous particle, to determine the drag force exerted by the flow on a particle, and incorporate the drag information into a macroscopic description of the packed bed of porous particles. Slow extensional flow past a sphere was studied by Bhatt[10]. Stokes flow past a permeable sphere for a non-axi-symmetric case was investigated by Padmavati et al.[11].

Giveler and Altobelli[12] performed experimental measurement of the effective viscosity for the steady flow through a wall bounded by a porous medium and concluded that $\left(\mu_{2} / \mu_{1}\right)$ varies from 5.1 to 10.9 . Dassios et al.[13] did a celebrated work by finding the solution of Stokes equation in spheroidal co-ordinates. Later Dassios et al.[14] used the above solution to study the problem of Stokes flow past spheroidal particle-in-cell models with Happel and Kuwabara boundary conditions, which has wide range of applications in problems concerning the flow through swarms of spheroidal particles.

Ochoa-Tapia and Whitaker $[15,16]$ studied the momentum transfer at the boundary between a porous medium and a homogeneous fluid theoretically and experimentally. They develop the appropriate jump condition for momentum transport within the framework of the method of volume averaging and they compare the theory with the experimental studies of Beavers and Joseph[6] and they explore the use of a variable porosity model as a substitute for the jump condition.

Many authors have used the stress jump boundary condition in various flow problems and reported significant changes in results. Kuznetsov[17, 18] used the stress jump boundary condition at the fluid-porous interface to discuss flow in channels partially filled with porous medium. The problem of Stokes flow inside a porous spherical shell was solved by Raja-Sekhar and Amarnath[20] by using Darcy's law for porous outside region and Stokes flow for inside region. The problem of slow extensional bounded flows past a porous sphere whose surface is stretching radially with slip at the surface has been studied by Bhatt and Shirley[21]. Datta and Deo[22] have studied the problem of Stokes flow with slip and Kuwabara boundary conditions and evaluated the drag force experienced by a rigid spheroid in a cell. Viscous flow past a porous sphere with an impermeable core was studied by Bhattacharya and Raja Shekhar[23]. They consider an arbitrary Stokes flow past a porous sphere within an impermeable core and Brinkman's model is used for the porous medium. The stress jump boundary condition for tangential stresses together with continuity of velocity components and continuity of normal stresses at the permeable boundary are used. They found that the drag and torque not only change with the change of the permeability, but also a significant effect of the stress jump co-efficient is observed. Also, they have used this boundary condition to discuss the arbitrary Stokes flow inside a porous spherical shell[24].

Srivastava and Srivastava[25] studied the Stokes flow through a porous sphere using stress jump condition at the fluid-porous interface and matching Stokes and Oseen's solutions far away from the sphere. They concluded that drag on a porous sphere decreases with increase of permeability of the medium. Deo and Yadav[26] examined flow past a swarm of porous deformed oblate spheroidal particles with kuwabara boundary condition and evaluated the drag force and studied the effects of deformation parameter on the drag force. Chandesris and Jamet[27] discuss the Boundary conditions at a planar fluid-porous. They have shown that, given a single volume-averaged transport equation in the entire domain (mesoscopic scale), it is possible to solve the problem analytically inside the heterogeneous transition zone using the method of matched asymptotic expansions and thus to analytically derive the boundary conditions that must be applied at the discontinuous interface. This analytical study shows that the stress jump condition is related to the slip velocity but also to the pressure gradient, through two jump parameters. This analysis also provides explicit relations between these two jump parameters and the variations of porosity and permeability in the transition zone through excess quantities. They, also discuss the Boundary conditions at fluid-porous interface[29]. They show that it is possible to provide an explicit relation between the values of jump parameters of the stress jump condition that one should impose at a fluid/porous interface and the structure of the transition 
region. An explicit relation between the jump parameters, the location of the discontinuous interface (macroscopic description) and the structure of the transition region (mesoscopic description) is obtained. This relation allows to explain the large sensitivity of the jump parameters to the location of the discontinuous interface observed in[27]. Diffusive mass transfer between a microporous medium and an homogeneous fluid was studies by Valdes-Parada et $\mathrm{al}[28]$. They obtain the jump boundary condition between a fluid and a porous medium, for Diffusive and chemical reaction. They, also discuss the Jump momentum boundary condition at a fluid-porous dividing surface[30]. They derived an expression for a jump stress boundary condition free of adjustable coefficient using a method of volume averaging method. The motivations of these papers lead us to discuss the present problem which includes the earlier results for the drag force on a porous sphere.

This paper concerns the problem of slow viscous flow through a swarm of clusters of porous spherical particles. As boundary conditions, continuity of velocity, continuity of normal stress and stress-jump condition at the porous and fluid interface, the continuity of velocity components on the solid spherical core are employed. On the hypothetical surface, uniform velocity and Happel boundary conditions are used. The drag force experienced by each porous spherical shell in a cell is evaluated. As a particular case, the drag force experienced by a porous sphere in a cell and in an unbounded medium with jump is also investigated. The earlier results reported for the drag force by Davis and Stone[9] for the drag force experienced by a porous sphere in a cell without jump, Happel[4] for a solid sphere in a cell and Qin and Kaloni[8] for a porous sphere in an unbounded medium have been then deduced.

\section{Statements and Mathematical Formulation of the Problem}

Here we have considered an axi-symmetric Stokes flow of a viscous incompressible fluid through a swarm of porous spherical particles of radius $b$ enclosing an impermeable spherical core of radius $a$. The above model is equivalent to a co-axial porous spherical shell enclosing the impermeable core. Further, we assume that, this porous shell is enveloped by a concentric sphere of radius $c(c>b)$, named as cell surface (Figure-2). The Stokes flow of a Newtonian fluid with absolute fluid viscosity is assumed to be steady and axi-symmetric. Let us consider that porous spherical shells are stationary and steady axi-symmetric viscous flow has been established around and through it by a uniform velocity $U$ directed in the positive z-axis. The radius $c$ of hypothetical cell is so chosen that the particle volume fraction $\gamma$ of the swarm is equal to the particle volume fraction of the cell, i.e. relative to this composite sphere (i.e. a core with porous shell) in the hypothetical cell

$$
\gamma=\frac{\pi b^{3}}{\pi c^{3}}
$$

The flow inside the porous shell region $\left(a<r^{*}<b\right)$, is governed by the Brinkman[1] equation

$$
\mu_{e} \nabla^{2} \mathbf{v}-\frac{\mu \mathbf{v}}{k}=\nabla p, \nabla \cdot \mathbf{v}=0,
$$

where, $k$ being the permeability of the porous medium, $\mathbf{V}$ and $p$ are the velocity vector and pressure at any point in the porous region, respectively. The coefficient $\mu_{e}$ is the effective viscosity for the Brinkman flow which taken to be different from $\mu$, the viscosity coefficient of clear fluid. The flow in the outside region of the porous spherical shell be governed by the Stokes equation (Happel and Brenner[31]) as

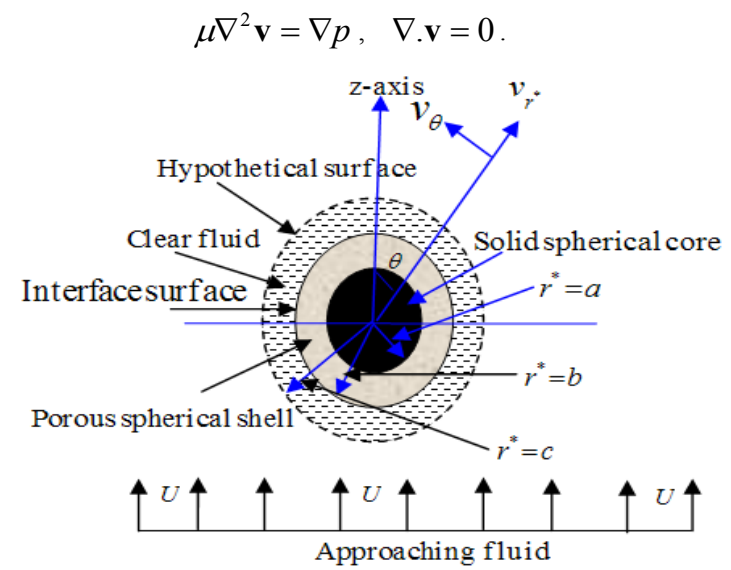

Figure 2. Physical situation and co-ordinate system of the problem

Let $v_{r^{*}}$ and $v_{\theta}$ be the velocity components in the direction of $r^{*}$ and $\theta$, respectively. Then the Stokes stream function $\psi$ which satisfies equation of continuity in spherical polar coordinates $(r, \theta, \varphi)$ can be expressed as

$$
v_{r^{*}}=-\frac{1}{\left(r^{*}\right)^{2} \sin \theta} \frac{\partial \psi}{\partial \theta}, v_{\theta}=\frac{1}{r^{*} \sin \theta} \frac{\partial \psi}{\partial r^{*}} .
$$

Also, let the index in the superscript under bracket of an entity $\chi^{(i)}, i=1,2$ indicates porous and clear fluid regions, respectively. Using the following variables

$$
\psi=U b^{2} \psi^{(i)}, p=\frac{\mu U}{b} p^{(i)}, r^{*}=b r, \zeta=\cos \theta
$$

and eliminating the pressures from both equations (2) and (3), we get the following fourth order partial differential equations, respectively as

$$
\begin{aligned}
& E^{2}\left(E^{2}-\alpha^{2}\right) \psi^{(1)}=0, \\
& E^{2}\left(E^{2} \psi^{(2)}\right)=0 .
\end{aligned}
$$

where, $\alpha^{2}=\sigma^{2} / \lambda^{2}$ with $\lambda^{2}=\mu_{e} / \mu, \quad \sigma^{2}=b^{2} / k \quad$ and $E^{2}$ being the dimensionless operator defined by

$$
E^{2}=\frac{\partial^{2}}{\partial r^{2}}+\frac{\left(1-\zeta^{2}\right)}{r^{2}} \frac{\partial^{2}}{\partial \zeta^{2}} .
$$

In the case of axi-symmetric incompressible creeping flow, the regular solution on the symmetry axis of Stokes equation (7) can be expressed (Happel \& Brenner[31]) as 


$$
\begin{aligned}
\psi^{(2)}(r, \zeta)= & \sum_{n=}^{\infty}\left[A_{n}^{(2)} r^{-n+1}+B_{n}^{(2)} r^{n}+C_{n}^{(2)} r^{-n+3}\right. \\
& \left.+D_{n}^{(2)} r^{n+2}\right] G_{n}(\zeta)
\end{aligned}
$$

The complete regular solution on symmetry axis of Brinkman's equation (6) may be expressed (Zlatanovski[19]) as

$$
\begin{aligned}
\psi^{(1)}(r, \zeta)=\sum_{n=2}^{\infty} & {\left[A_{n}^{(1)} r^{-n+1}+B_{n}^{(1)} r^{n}+C_{n}^{(1)} y_{-n}(\alpha r)\right.} \\
& \left.+D_{n}^{(1)} y_{n}(\alpha r)\right] G_{n}(\zeta)
\end{aligned}
$$

In order to finalize the proposed boundary value problem, we should impose appropriate boundary conditions which are physically realistic and mathematically consistent for this proposed problem. No slip boundary conditions are imposed on the surface of solid spherical core:

$$
v_{r^{*}}^{(1)}(r, \theta)=0 \text { and } v_{\theta}^{(1)}(r, \theta)=0 \text { at } r^{*}=a .
$$

On the boundary between the porous shell and the liquid $r^{*}=b$, we assume the velocity components and normal stresses $T_{r^{*} r^{*}}(r, \theta)$ are continuous:

$$
\begin{gathered}
v_{r^{*}}^{(1)}(r, \theta)=v_{r^{*}}^{(2)}(r, \theta), v_{\theta}^{(1)}(r, \theta)=v_{\theta}^{(2)}(r, \theta) \\
T_{r^{*} r^{*}}^{(1)}(r, \theta)=T_{r^{*} r^{*}}^{(2)}(r, \theta) .
\end{gathered}
$$

Using the continuity of velocity components (12), the boundary condition suggested by Ochao-Tapia and Whitaker[15, 16] at the interface of porous shell and clear fluid $r^{*}=b$, will become as (Srivastava and Srivastava[25]):

$$
T_{r^{*} \theta}^{(1)}(r, \theta)-T_{r^{*} \theta}^{(2)}(r, \theta)=\frac{\beta \mu}{\sqrt{k}} v_{\theta}^{(1)}(r, \theta) .
$$

The above condition is named as stress jump boundary condition for the tangential stress.

Here, it may be mentioned that the values of jump coefficient $\beta$ varies in the range -1 to 1 as experimentally found by Ochoa-Tapia and Whitaker $[15,16]$. If $\beta=0$, then we get the continuity of shearing stress.

The continuity of the redial components of fluid velocity on the outer cell $\left(r^{*}=c\right)$ implies:

$$
v_{r^{*}}^{(2)}(r, \theta)=U \cos \theta \text {. }
$$

According to Happel[4] the tangential stress vanishes on the outer cell surface $r^{*}=c$ :

$$
T_{r^{*} \theta}^{(2)}(r, \theta)=0 .
$$

It may be noted here that, for the case of flow past a sphere, when we apply the boundary conditions (11) - (16), all the coefficients appearing in the stream functions (9) and (10) vanishes except for $n=2$, (Datta and Deo[22]).

Therefore, a particular regular solution of the Brinkman equation (6) can be expressed as

$$
\psi^{(1)}(r, \zeta)=\left[A_{1} r^{2}+\frac{B_{1}}{r}+C_{1} y_{-2}(\alpha r)+D_{1} y_{2}(\alpha r)\right] G_{2}(\zeta)
$$

and a particular regular solution of the Stokes equation may be taken as

$$
\psi^{(2)}(r, \zeta)=\left[A_{2} r^{2}+\frac{B_{2}}{r}+C_{2} r+D_{2} r^{4}\right] G_{2}(\zeta) .
$$

Here, $\quad y_{-2}(\alpha r)=\alpha \sinh (\alpha r)-(1 / r) \cosh (\alpha r)$ $y_{2}(\alpha r)=\alpha \cosh (\alpha r)-(1 / r) \sinh (\alpha r)$,
$G_{2}(\zeta)=(1 / 2)\left(1-\zeta^{2}\right)$ and $A_{i}, B_{i}, C_{i}, D_{i}(i=1,2)$ are arbitrary constant which we have to be determined.

\subsection{Determination of Arbitrary Constants}

As a result of application of the boundary conditions (11) - (16) and solving the resulting equations, we get the values of arbitrary constants $A_{1}, B_{1}, C_{1}, D_{1}, A_{2}, B_{2}, C_{2}$ and $D_{2}$, which are given in the Appendix A. Thus, all the coefficients have been determined and hence, we get the explicit expressions for stream functions from equations (17) and (18), in both regions.

\section{Evaluation of Drag force}

The drag force experienced by each porous spherical shell in a cell can be evaluated by using the simple elegant formula (Happel and Brenner[31], p.-115) as

$$
F=\pi \mu U b \int_{0}^{\pi} \varpi^{3} \frac{\partial}{\partial r}\left(\frac{E^{2} \psi^{(1)}}{\varpi^{2}}\right) r d \theta .
$$

Here, since $\varpi=r \sin \theta$ and

$$
E^{2} \psi^{(1)}=\left[-\frac{2}{r} C_{2}+10 r^{2} D_{2}\right] G_{2}(\zeta),
$$

inserting these above values in (19) and integrating, we find that

$$
F=4 \pi \mu b U C_{2},
$$

where, $C_{2}$ is given in appendix A. Here, it may be noted that Stokeslet coefficient $C_{2}$ of the stream function only contribute to the drag force.

Also, the drag coefficient $C_{D}$ can be defined as

$$
\begin{aligned}
C_{D} & =\frac{F}{(1 / 2) \rho U^{2} \pi b^{2}} \\
& =\frac{16 C_{2}}{\operatorname{Re}},
\end{aligned}
$$

where, $\operatorname{Re}=\frac{2 b U}{v}$ and $v=\frac{\mu}{\rho}$ being the Reynolds number and kinematic viscosity of fluid, respectively.

When hypothetical cell radius $c \rightarrow \infty$ i.e., $m \rightarrow \infty$, then the porous spherical shell lies in an unbounded medium. In this case, the value of the drag force comes out as

$$
F=12 \pi \mu U b C_{2}^{*}
$$

where, $C_{2}^{*}$ is given in Appendix B.

This is a new result reported for the drag force experienced by a porous spherical shell in an unbounded medium with jump condition.

In the limit of $a \rightarrow 0$, i.e., $\ell \rightarrow 0$, the porous spherical shell reduces to the porous sphere in a cell. Therefore, the value of drag force $F$ experienced by the porous sphere of radius $b$ comes out as

$$
F=4 \pi \mu b U C_{3}^{*}
$$

where, $C_{3}^{*}$ is given in Appendix B

This is also a new result reported for the drag force experienced by a porous sphere in a cell with jump condition.

Also, in the limit $a \rightarrow 0$ i.e., $\ell \rightarrow 0$ and $m \rightarrow \infty$, the physical problems corresponds to the porous sphere of radius 
$b$ in unbounded medium. In this case, the value of drag force $F$ experienced by a porous sphere comes out as

$$
\begin{aligned}
F= & 3 \alpha \lambda\left[\alpha\left\{\beta\left(12+\left(-12+\alpha^{2}\right) \lambda^{2}\right)+\alpha \lambda\left(-6+\left(6+\alpha^{2}\right) \lambda^{2}\right)\right\}\right. \\
& \cosh \alpha-\left\{4\left(3+\alpha^{2}\right) \beta-2 \alpha\left(3+\alpha^{2}\right) \lambda+\left(-12-3 \alpha^{2}+\alpha^{4}\right)\right. \\
& \left.\left.\beta \lambda^{2}+3 \alpha\left(2+\alpha^{2}\right) \lambda^{3}\right\} \sinh \alpha\right] / \Delta_{3} \\
\text { where, } & \\
\Delta_{3}= & {\left[\alpha \left(-54+\lambda\left(54 \lambda+\alpha\left(\beta\left(27+2\left(-12+\alpha^{2}\right) \lambda^{2}\right)+\alpha \lambda\right.\right.\right.\right.} \\
& \left.\left.\left.\left(-9+2\left(6+\alpha^{2}\right) \lambda^{2}\right)\right)\right)\right) \cosh \alpha-\left(-18\left\{3+\alpha^{2}+9 \alpha(3\right.\right. \\
& \left.+\alpha^{2}\right) \beta \lambda-3\left(-18-3 \alpha^{2}+2 \alpha^{4}\right) \lambda^{2}+2 \alpha\left(-12-3 \alpha^{2}\right. \\
& \left.\left.\left.+\alpha^{4}\right) \beta \lambda^{3}+6 \alpha^{2}\left(2+\alpha^{2}\right) \lambda^{4}\right) \sinh \alpha\right] .
\end{aligned}
$$

The following special cases can be deduced from the present analysis:

I. Drag on a porous sphere in a cell without $\operatorname{jump}(\beta=0)$ :

If $a \rightarrow 0$, i.e., $\ell \rightarrow 0$, then the porous spherical shell reduces to the porous sphere of radius $b$. In this case, the value of drag force $F$ experienced by a porous sphere for the case of $\lambda=1$ comes out as

$$
F=\frac{\left.-3\left(\alpha^{2}+4 \alpha^{2} \lambda_{1}^{5}+10 \lambda_{1}^{5}\right) \alpha^{-1} \sinh \alpha\right]}{\Delta_{4}},
$$

where,

$$
\begin{aligned}
\Delta_{4}= & {\left[2 \alpha^{2}-3 \alpha^{2} \lambda_{1}+3 \alpha^{2} \lambda_{1}^{5}-2 \alpha^{2} \lambda_{1}^{6}+3+42 \lambda_{1}^{5}-30 \lambda_{1}^{6}\right.} \\
& \left.+90 \alpha^{-2} \lambda_{1}^{5}\right] \cosh \alpha-\left[-3 \alpha^{2} \lambda_{1}+15 \alpha^{2} \lambda_{1}^{5}-12 \alpha^{2} \lambda_{1}^{6}\right. \\
& \left.+3+72 \lambda_{1}^{5}-30 \lambda_{1}^{6}+90 \alpha^{-2} \lambda_{1}^{5}\right] \alpha^{-1} \sinh \alpha \\
\text { and } \lambda_{1}= & \frac{1}{m} .
\end{aligned}
$$

This agrees with the result of Davis and Stone[9] for the drag force experienced by a porous sphere in a cell.

II. Drag on a porous sphere in an unbounded medium without jump $(\beta=0)$ :

If $a \rightarrow 0$ i.e., $\ell \rightarrow 0$ and $m \rightarrow \infty$, then the porous spherical shell reduces to the porous sphere in an unbounded medium. In this case, the value of drag force $F$ experienced by a porous sphere of radius $b$ for the case of

$$
\lambda=1 \text { turns out as }
$$

$$
F=6 \pi \mu b U\left[1+\lambda_{2}+\frac{3}{2 \alpha^{2}}\right]^{-1}, \lambda_{2}=\sinh \alpha /(\alpha \cosh \alpha-\sinh \alpha) .
$$

This agrees with the result of Qin and Kaloni[8] for the drag force experienced by a porous sphere in an unbounded medium.

\section{Drag on a solid sphere in a cell:}

In this case, the value of the drag force from equation (27) by taking limit as $\alpha \rightarrow \infty$, reduces to

$$
F=6 \pi \mu U b\left[1+\frac{2}{3} \gamma^{5 / 3}\right]\left[1-\frac{3}{2} \gamma^{1 / 3}+\frac{3}{2} \gamma^{5 / 3}-\gamma^{2}\right]^{-1} .
$$

This agrees with the result for the drag force as reported earlier by Happel[4].

\section{Drag on a solid sphere in an unbounded medium:}

If $c \rightarrow \infty$ i.e. $\gamma \rightarrow 0$, then from equation (30), the drag force experienced by solid sphere of radius $b$ in an unbounded medium is comes out as

$$
F=6 \pi \mu U b .
$$

A well known result celebrated by Stokes for the drag experienced by a solid sphere in an unbounded medium.

\section{Results and Discussions}

In this study, the drag force experienced by a porous spherical shell in a cell, porous spherical shell in an unbounded medium, porous sphere in a cell and porous sphere in an unbounded medium with jump are evaluated. The dependence of the drag coefficient $C_{D}$ on various parameters like permeability parameter $\sigma$, viscosity ratio $\lambda$, particle volume fraction $\gamma$, porosity parameter $\lambda$ and jump coefficient $\beta$ are discussed. The variation of $\operatorname{Re} C_{D}$ versus particle volume fraction $\gamma$ and permeability parameter $\sigma$ for the porous spherical shell is shown in Fig.-3. It is observed that $\operatorname{Re} C_{D}$ slightly increases with increase in permeability parameter $\sigma$ for low values of particle volume fraction $\gamma$ $(\gamma<0.3)$ as for small values of $\gamma$ the flow around a shell is not influenced very much by neighbouring particles. However, for large values of particle volume fraction $\gamma$ $(\gamma>0.3)$ a significant increase in $\operatorname{Re} C_{D}$ is observed with $\sigma$ except for very low values of permeability parameter $\sigma$ $(\sigma<3)$.

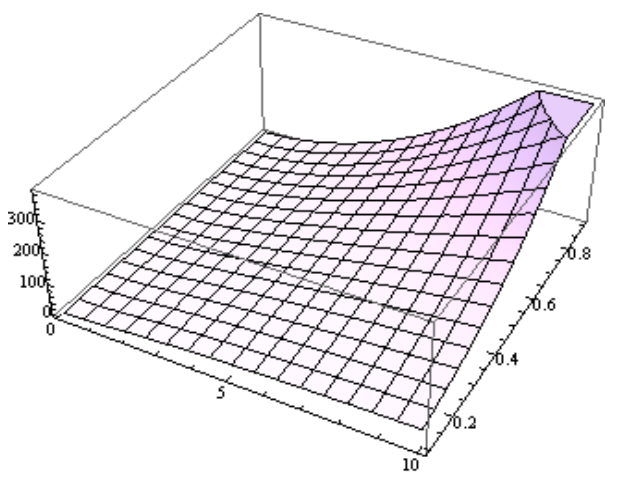

Figure 3. Variation of $\mathrm{ReC}_{\mathrm{D}}$ versus particle volume fraction $\boldsymbol{\gamma}$ and permeability parameter $\sigma$ for the porous spherical shell when porosity parameter $\lambda=2$, jump coefficient $\beta=0.3$ and $\ell=0.5$

The dependence of $\operatorname{Re} C_{D}$ on permeability parameter $\sigma$ and jump coefficient $\beta$ is shown in Fig.-4. For all values of jump coefficient $\beta, \operatorname{Re} C_{D}$ asymptotically increases with increase of permeability parameter $\sigma$. However, $\operatorname{Re} C_{D}$ gradually decreases with increase in jump coefficient $\beta$ for small values of permeability parameter $\sigma$ and for large values of $\sigma, \operatorname{Re} C_{D}$ asymptotically decreases. For all values of jump coefficient $\beta, \operatorname{Re} C_{D}$ increases with particle volume fraction $\gamma$ (Fig.-5). It is also fined out that $\operatorname{Re} C_{D}$ gradually decreases with increase in jump coefficient $\beta$ for all values of $\gamma$. It clearly indicates that the variation of $\operatorname{Re} C_{D}$ with particle volume fraction $\gamma$ is independent of the shearing stress in the clear fluid relative to the shearing stress in the porous region at the fluid-porous interface. Here, as $\beta$ 
increases the value of $\operatorname{Re} C_{D}$ decreases. Hence, it is felt that there is a significant effect of the stress jump coefficient $\beta$ on the flow like volume flow, drag force etc. Therefore while studying viscous flow problems involving Stokes equation in clear fluid region and Brinkman equation in porous media; one has to take the stress jump in the tangential stress components into consideration, which has a significant impact on the physical problem. Also, this helps us to estimate the flow quantities more accurately and to realize the dependency of the physical problem on the boundary conditions.
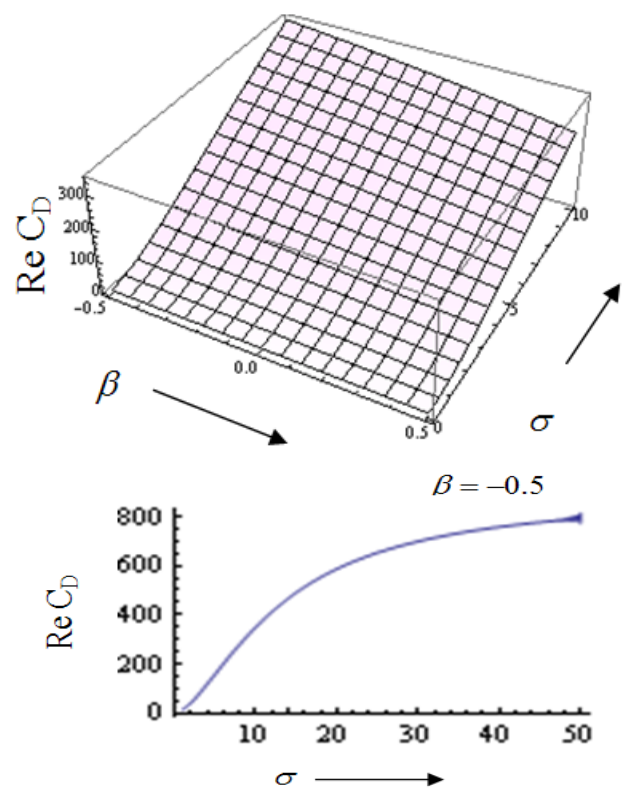

Figure 4. Variation of $\mathrm{ReC}_{\mathrm{D}}$ versus jump coefficient $\beta$ and permeability parameter $\sigma$ for the porous spherical shell when porosity parameter $\lambda=2$, particle volume fraction $\gamma=0.5$ and $\ell=0.5$
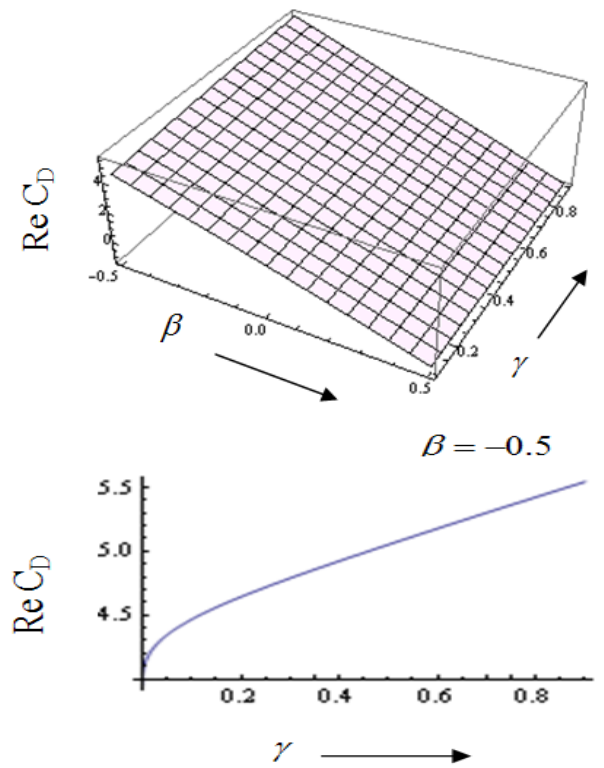

Figure 5. Variation of $\operatorname{ReC}_{\mathrm{D}}$ versus jump coefficient $\beta$ and particle volume fraction $\gamma$ for the porous spherical shell when porosity parameter $\lambda=2$, permeability parameter $\sigma=0.5$ and $\ell=0.5$

The variation of $\operatorname{Re} C_{D}$ with particle volume fraction $\gamma$ and porosity parameter $\lambda$ is shown in Fig.-6. We observe that $\operatorname{Re} C_{D}$ decreases with increase in particle volume fraction $\gamma$ for small porosity parameter $\lambda(\lambda<5)$. As the porosity parameter increases $(\lambda>5), \operatorname{Re} C_{D}$ significantly increases to an asymptotic value with particle volume fraction. Evidently, we conclude that higher the effective viscosity in the porous medium comparative to the viscosity of the clear fluid, higher will be the rate of increase of drag force with particle volume fraction $\gamma$ at the fluid-porous interface.

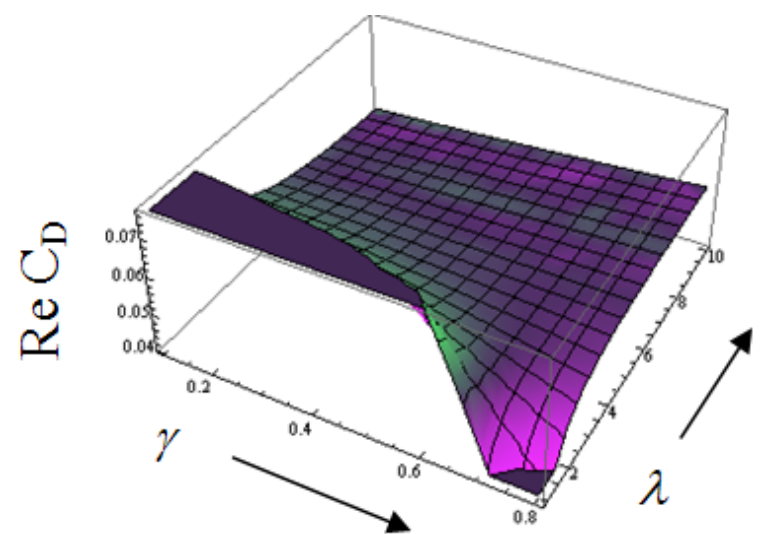

Figure 6. Variation of $\mathrm{ReC}_{\mathrm{D}}$ versus porosity parameter $\lambda$ and particle volume fraction $\gamma$ for the porous spherical shell when jump coefficient $\beta=0.8$, permeability parameter $\sigma=0.1$ and $\ell=0.5$

In Fig.-7, the dependence of $\operatorname{Re} C_{D}$ on porosity parameter $\lambda$ and permeability parameter $\sigma$ is shown. The term $\operatorname{Re} C_{D}$ increases to an asymptotic value with permeability parameter $\sigma$. For all values of permeability parameter $\operatorname{Re} C_{D}$ first increases rapidly with porosity parameter $\lambda$ and then become steady. Here the numerical results and figures for given input values have been evaluated through Mathematica software.

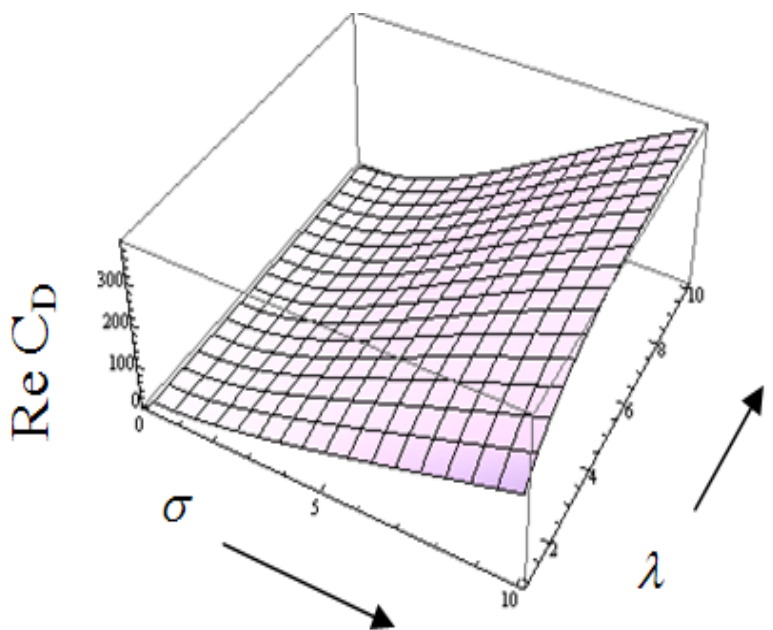

Figure 7. Variation of $\mathrm{ReC}_{\mathrm{D}}$ versus porosity parameter $\lambda$ and permeability parameter $\sigma$ for the porous spherical shell when jump coefficient $\beta=0.8$, particle volume fraction $\gamma=0.5$ and $\ell=0.5$

\section{Appendix A}


$C_{2}=m \alpha \lambda\left[y_{2}(\alpha \ell)\left\{y_{-2}(\alpha)\left(\beta\left(72+48 \lambda^{2}-4 \alpha^{2} \lambda^{2}-6 \ell^{3} \alpha^{2} \lambda^{2}\right.\right.\right.\right.$ $+4 \ell^{4}\left(-18+\left(-12+\alpha^{2}\right) \lambda^{2}\right)+m^{5}\left(-72-9 \ell^{3} \alpha^{2} \lambda^{2}-6\left(12+\alpha^{2}\right.\right.$ )$\left.\left.\lambda^{2}+6 \ell^{4}\left(12+\left(-12+\alpha^{2}\right) \lambda^{2}\right)\right)\right)+\alpha \lambda\left(3 m^{5}\left(-2\left(-6+\left(6+\alpha^{2}\right)\right.\right.\right.$ $\left.\left.\lambda^{2}\right)+2 \ell^{4}\left(-6+\left(6+\alpha^{2}\right) \lambda^{2}\right)-3 \ell^{3}\left(-18+\left(18+\alpha^{2}\right) \lambda^{2}\right)\right)+2($ $-2\left(9+\left(6+\alpha^{2}\right) \lambda^{2}\right)+2 \ell^{4}\left(9+\left(6+\alpha^{2}\right) \lambda^{2}\right)-3 \ell^{3}(27+((18+$ $\left.\left.\left.\left.\left.\alpha^{2}\right) \lambda^{2}\right)\right)\right)\right)+\alpha^{2}\left(-12 y_{-1}(\alpha \ell) \ell^{2}(2 \beta-\alpha \lambda)\left(3+2 \lambda^{2}+3 m^{5}(-1\right.\right.$ $\left.\left.+\lambda^{2}\right)\right)+y_{-1}(\alpha)\left(-2\left(-2-3 \ell^{3}+2 \ell^{4}\right) \alpha \lambda\left(3+2 \lambda^{2}+3 m^{5}(-1+\right.\right.$ $\left.\left.\lambda^{2}\right)\right)+\beta\left(4\left(-6+\left(-4+\alpha^{2}\right) \lambda^{2}\right)-4 \ell^{4}\left(-6+\left(-4+\alpha^{2}\right) \lambda^{2}\right)+6\right.$ $\ell^{3}\left(12+\left(8+\alpha^{2}\right) \lambda^{2}\right)-3 m^{5}\left(-8-2\left(-4+\alpha^{2}\right) \lambda^{2}+2 \ell^{4}(4+(-4\right.$ $\left.\left.\left.\left.\left.\left.\left.+\alpha^{2}\right) \lambda^{2}\right)-3 \ell^{3}\left(-8+\left(8+\alpha^{2}\right) \lambda^{2}\right)\right)\right)\right)\right)\right\}+y_{-2}(\alpha \ell)\left\{y_{2}(\alpha)(\beta(-\right.$ $72-48 \lambda^{2}+4 \alpha^{2} \lambda^{2}+6 \ell^{3} \alpha^{2} \lambda^{2}+\ell^{4}\left(72-4\left(-12+\alpha^{2}\right) \lambda^{2}\right)+m^{5}$ $\left.\left(72+9 \ell^{3} \alpha^{2} \lambda^{2}+6\left(-12+\alpha^{2}\right) \lambda^{2}-6 \ell^{4}\left(12+\left(-12+\alpha^{2}\right) \lambda^{2}\right)\right)\right)$ $+\alpha \lambda\left(4\left(9+\left(6+\alpha^{2}\right) \lambda^{2}\right)-4 \ell^{4}\left(9+\left(6+\alpha^{2}\right) \lambda^{2}\right)+6 \ell^{3}(27+(18\right.$ $\left.\left.+\alpha^{2}\right) \lambda^{2}\right)+m^{5}\left(6\left(-6+\left(6+\alpha^{2}\right) \lambda^{2}\right)-6 \ell^{4}\left(-6+\left(6+\alpha^{2}\right) \lambda^{2}\right)+9\right.$ $\left.\left.\left.\ell^{3}\left(-18+\left(18+\alpha^{2}\right) \lambda^{2}\right)\right)\right)\right)+\alpha^{2}\left(12 y_{1}(\alpha \ell) \ell^{2}(2 \beta-\alpha \lambda)\left(3+2 \lambda^{2}\right.\right.$ $\left.+3 m^{5}\left(-1+\lambda^{2}\right)\right)+y_{1}(\alpha)\left(2\left(-2-3 \ell^{3}+2 \ell^{4}\right) \alpha \lambda\left(3+2 \lambda^{2}+3 m^{5}\right.\right.$ $\left.\left(-1+\lambda^{2}\right)\right)+\beta\left(3 m^{5}\left(-8-2\left(-4+\alpha^{2}\right) \lambda^{2}+2 \ell^{4}\left(4+\left(-4+\alpha^{2}\right) \lambda^{2}\right.\right.\right.$ )$\left.-3 \ell^{3}\left(-8+\left(8+\alpha^{2}\right) \lambda^{2}\right)\right)+2\left(12-2\left(-4+\alpha^{2}\right) \lambda^{2}+2 \ell^{4}(-6+\right.$ $\left.\left.\left.\left.\left.\left.\left(-4+\alpha^{2}\right) \lambda^{2}\right)-3 \ell^{3}\left(12+\left(8+\alpha^{2}\right) \lambda^{2}\right)\right)\right)\right)\right)\right\}+\ell \alpha^{2}\left\{4\left(y_{-2}(\alpha) y_{1}\right.\right.$ $\left.(\alpha)-y_{-1}(\alpha) y_{2}(\alpha)\right)+\ell\left(2+\ell^{4}\right)(2 \beta-\alpha \lambda)\left(3+2 \lambda^{2}+3 m^{5}(-1+\right.$ $\left.\left.\lambda^{2}\right)\right)+y_{1}(\alpha \ell)\left(-y_{-1}(\alpha) \alpha^{2}\left(2\left(2+\ell^{3}\right) \alpha \lambda\left(3+2 \lambda^{2}+3 m^{5}\left(-1+\lambda^{2}\right.\right.\right.\right.$ )$+\beta\left(3 m^{5}\left(8+2\left(-4+\alpha^{2}\right) \lambda^{2}+\ell^{3}\left(-8+\left(8+\alpha^{2}\right) \lambda^{2}\right)\right)+2(2(-\right.$ $\left.\left.\left.\left.6+\left(-4+\alpha^{2}\right) \lambda^{2}\right)+\ell^{3}\left(12+\left(8+\alpha^{2}\right) \lambda^{2}\right)\right)\right)\right\}+y_{-2}(\alpha)\{\beta(-72+$ $\left.2\left(-24+\left(2+\ell^{3}\right) \alpha^{2}\right) \lambda^{2}+3 m^{5}\left(24+\left(-24+\left(2+\ell^{3}\right) \alpha^{2}\right) \lambda^{2}\right)\right)+\alpha$ $\lambda\left(4\left(9+\left(6+\alpha^{2}\right) \lambda^{2}\right)+2 \ell^{3}\left(27+\left(18+\alpha^{2}\right) \lambda^{2}\right)+3 m^{5}(2(-6+(6\right.$ $\left.\left.\left.\left.\left.\left.+\alpha^{2}\right) \lambda^{2}\right)+\ell^{3}\left(-18+\left(18+\alpha^{2}\right) \lambda^{2}\right)\right)\right)\right)\right)+y_{-1}(\alpha \ell)\left(-y_{1}(\alpha) \alpha^{2}(2\right.$ $\left(2+\ell^{3}\right) \alpha \lambda\left(3+2 \lambda^{2}+3 m^{5}\left(-1+\lambda^{2}\right)\right)+\beta\left(3 m^{5}\left(8+2\left(-4+\alpha^{2}\right) \lambda^{2}\right.\right.$ $\left.+\ell^{3}\left(-8+\left(8+\alpha^{2}\right) \lambda^{2}\right)\right)+2\left(2\left(-6+\left(-4+\alpha^{2}\right) \lambda^{2}\right)+\ell^{3}(12+(8+\right.$ $\left.\left.\left.\left.\left.\alpha^{2}\right) \lambda^{2}\right)\right)\right)\right)+y_{2}(\alpha)\left\{\beta\left(-72+2\left(-24+\left(2+\ell^{3}\right) \alpha^{2}\right) \lambda^{2}+3 m^{5}(24\right.\right.$ $\left.\left.+\left(-24+\left(2+\ell^{3}\right) \alpha^{2}\right) \lambda^{2}\right)\right)+\alpha \lambda\left(4\left(9+\left(6+\alpha^{2}\right) \lambda^{2}\right)+2 \ell^{3}(27+(1\right.$ $\left.\left.8+\alpha^{2}\right) \lambda^{2}\right)+3 m^{5}\left(2\left(-6+\left(6+\alpha^{2}\right) \lambda^{2}\right)+\ell^{3}\left(-18+\left(18+\alpha^{2}\right) \lambda^{2}\right)\right.$ )$))\}\}] / \Delta$

$\Delta=\ell \alpha^{2}\left[-2\left\{y_{-2}(\alpha) y_{1}(\alpha)-y_{-1}(\alpha) y_{2}(\alpha)\right) \ell\left(2+\ell^{4}\right)\left(-6 m^{5}\right.\right.$ $\alpha \lambda(-2 \beta+\alpha \lambda)\left(-1+\lambda^{2}\right)-2 \alpha \lambda(-2 \beta+\alpha \lambda)\left(3+2 \lambda^{2}\right)+m^{6}$ $\left(\alpha \beta \lambda\left(9-8 \lambda^{2}\right)+18\left(-1+\lambda^{2}\right)+\alpha^{2} \lambda^{2}\left(-3+4 \lambda^{2}\right)\right)+3 m(6$ $\left.\left.+4 \lambda^{2}+\alpha \beta\left(2 \lambda-4 \lambda^{3}\right)+\alpha^{2}\left(\lambda^{2}+2 \lambda^{4}\right)\right)\right)+y_{1}(\alpha \ell)\left(2 m^{6}\right.$ $\left(y_{-2}(\alpha)\left(\left(2+\ell^{3}\right) \alpha^{3} \beta \lambda^{3}+\left(2+\ell^{3}\right) \alpha^{4} \lambda^{4}+54\left(-1+\lambda^{2}\right)-3 \alpha\right.\right.$ $\left.\left.\beta \lambda\left(-9+8 \lambda^{2}\right)+3 \alpha^{2} \lambda^{2}\left(-3+4 \lambda^{2}\right)+6 \ell^{3}\left(-1+\lambda^{2}\right)\right)\right)-y_{-1}$ $(\alpha) \alpha^{2}\left(2 \alpha^{3} \beta \lambda^{3}+\alpha \beta \lambda\left(9-8 \lambda^{2}\right)+18\left(-1+\lambda^{2}\right)+\alpha^{2}\left(-6 \lambda^{2}\right.\right.$ $\left.+4 \lambda^{4}\right)+\ell^{3}\left(\alpha^{3} \beta \lambda^{3}-18\left(-1+\lambda^{2}\right)+\alpha^{2} \lambda^{2}\left(-3+4 \lambda^{2}\right)+\alpha \beta \lambda\right.$ $\left.\left.\left.\left(-9+8 \lambda^{2}\right)\right)\right)\right)-3 m^{5} \alpha \lambda\left(y_{-1}(\alpha) \alpha^{2}\left(2\left(2+\ell^{3}\right) \alpha \lambda\left(-1+\lambda^{2}\right)+\right.\right.$ $\left.\beta\left(8+2\left(-4+\alpha^{2}\right) \lambda^{2}+\ell^{3}\left(-8+\left(8+\alpha^{2}\right) \lambda^{2}\right)\right)\right)+y_{-2}(\alpha)(\beta($ $\left.24+\left(-24+\left(2+\ell^{3}\right) \alpha^{2}\right) \lambda^{2}\right)+\alpha \lambda\left(2\left(-6+\left(6+\alpha^{2}\right) \lambda^{2}\right)+\ell^{3}\right.$ $\left.\left.\left.\left(-18+\left(18+\alpha^{2}\right) \lambda^{2}\right)\right)\right)\right)+2 \alpha \lambda\left(y_{-1}(\alpha) \alpha^{2}\left(\left(2+\ell^{3}\right) \alpha \lambda(-3\right.\right.$ $\left.+2 \lambda^{2}\right)+\beta\left(2\left(-6+\left(-4+\left(2+\ell^{3}\right) \alpha^{4} \lambda^{4}+12 \alpha \beta \lambda\left(1-2 \lambda^{2}\right)\right.\right.\right.$ $\left.\left.+2 \alpha^{2} \lambda^{2}\left(8+\ell^{3}\right)+6 \lambda^{2}+9 \ell^{3} \lambda^{2}\right)\right)-y_{-1}(\alpha) \alpha^{2}\left(2\left(6+4 \lambda^{2}\right)\right.$ $\left.+\alpha^{3} \beta \lambda^{3}+\alpha \beta\left(2 \lambda-4 \lambda^{3}\right)+2 \alpha^{2}\left(\lambda^{2}+\lambda^{4}\right)\right)+2 \alpha^{2}\left(\lambda^{2}+\lambda^{4}\right.$ )$))))-y_{-1}(\alpha \ell)\left(2 m^{6}\left(y_{2}(\alpha)\left(\alpha^{3} \beta \lambda^{3}\left(2+\ell^{3}\right)+\left(2+\ell^{3}\right) \alpha^{4} \lambda^{4}\right.\right.\right.$ $+54\left(-1+\lambda^{2}\right)-3 \alpha \beta \lambda\left(-9+8 \lambda^{2}\right)+3 \alpha^{2} \lambda^{2}\left(-3+4 \lambda^{2}\right)+6$ $\left.\left.\ell^{3}\left(-1+\lambda^{2}\right)\right)\right)-y_{1}(\alpha) \alpha^{2}\left(2 \alpha^{3} \beta \lambda^{3}+\alpha \beta \lambda\left(9-8 \lambda^{2}\right)+18(-\right.$ $\left.1+\lambda^{2}\right)+\alpha^{2}\left(-6 \lambda^{2}+4 \lambda^{4}\right)+\ell^{3}\left(\alpha^{3} \beta \lambda^{3}-18\left(-1+\lambda^{2}\right)+\alpha^{2}\right.$ $\left.\left.\left.\lambda^{2}\left(-3+2 \lambda^{2}\right)+\alpha \beta \lambda\left(-9+8 \lambda^{2}\right)\right)\right)\right)-3 m^{5} \alpha \lambda\left(-y_{1}(\alpha) \alpha^{2}(\right.$ $2\left(2+\ell^{3}\right) \alpha \lambda\left(-1+\lambda^{2}\right)+\beta\left(8+2\left(-4+\alpha^{2}\right) \lambda^{2}+\ell^{3}(-8+\right.$ $\left.\left.\left.\left(8+\alpha^{2}\right) \lambda^{2}\right)\right)\right)+y_{2}(\alpha)\left(\beta\left(24+\left(-24+\left(2+\ell^{3}\right) \alpha^{2}\right) \lambda^{2}\right)+\alpha\right.$ $\left.\left.\lambda\left(2\left(-6+\left(6+\alpha^{2}\right) \lambda^{2}\right)+\ell^{3}\left(-18+\left(18+\alpha^{2}\right) \lambda^{2}\right)\right)\right)\right)+2 \alpha \lambda$ $\left(y_{1}(\alpha) \alpha^{2}\left(\left(2+\ell^{3}\right) \alpha \lambda\left(3+2 \lambda^{2}\right)+\beta\left(2\left(-6+\left(-4+\alpha^{2}\right) \lambda^{2}\right)\right.\right.\right.$ $\left.\left.+\ell^{3}\left(12+\left(8+\alpha^{2}\right) \lambda^{2}\right)\right)\right)-y_{2}(\alpha)\left(\beta\left(-36+\left(-24+\left(2+\ell^{3}\right)\right.\right.\right.$ $\left.\left.\left.\alpha^{2}\right) \lambda^{2}+\alpha \lambda\left(2\left(9+\left(6+\alpha^{2}\right) \lambda^{2}\right)+\ell^{3}\left(27+\left(18+\alpha^{2}\right) \lambda^{2}\right)\right)\right)\right)$ $+3 m\left(y_{2}(\alpha)\left(36+24 \lambda^{2}+\left(2+\ell^{3}\right) \alpha^{3} \beta \lambda^{3}+\left(2+\ell^{3}\right) \alpha^{4} \lambda^{4}+\right.\right.$ $\left.\left.12 \alpha \beta \lambda\left(1-2 \lambda^{2}\right)+2 \alpha^{2} \lambda^{2}\left(8+\ell^{3}\right)+6 \lambda^{2}+9 \ell^{3} \lambda^{2}\right)\right)-y_{1}(\alpha)$ $\alpha^{2}\left(2\left(6+4 \lambda^{2}\right)+\alpha^{3} \beta \lambda^{3}+\alpha \beta\left(2 \lambda-4 \lambda^{3}\right)+2 \alpha^{2}\left(\lambda^{2}+\lambda^{4}\right)\right)$ $+\ell^{3}\left(\alpha^{3} \beta \lambda^{3}-4\left(3+2 \lambda^{2}\right)+\alpha \beta\left(-4 \lambda+8 \lambda^{3}\right)+2 \alpha^{2}\left(\lambda^{2}+\lambda^{4}\right.\right.$ )$))))+y_{2}(\alpha \ell)\left(3 m^{5} \alpha \lambda\left(y_{-2}(\alpha)\left(\beta\left(24+3 \ell^{3} \alpha^{2} \lambda^{2}+2(-12+\right.\right.\right.\right.$ $\left.\left.\alpha^{2}\right) \lambda^{2}-2 \ell^{4}\left(12+\left(-12+\alpha^{2}\right) \lambda^{2}\right)\right)+\alpha \lambda\left(2\left(-6+\left(6+\alpha^{2}\right) \lambda^{2}\right.\right.$ )$\left.\left.-2 \ell^{4}\left(-6+\left(6+\alpha^{2}\right) \lambda^{2}\right)+3 \ell^{3}\left(-18+\left(18+\alpha^{2}\right) \lambda^{2}\right)\right)\right)+\alpha^{2}$ $\left(12 y_{-1}(\alpha \ell) \ell^{2}(2 \beta-\alpha \lambda)\left(-1+\lambda^{2}\right)+y_{-1}(\alpha)\left(2\left(-2-3 \ell^{3}+2\right.\right.\right.$ $\left.\ell^{4}\right) \alpha \lambda\left(-1+\lambda^{2}\right)+\beta\left(-8-2\left(-4+\alpha^{2}\right) \lambda^{2}+2 \ell^{4}(4+(-4+\right.$ $\left.\left.\left.\left.\left.\left.\alpha^{2}\right) \lambda^{2}\right)-3 \ell^{3}\left(-8+\left(8+\alpha^{2}\right) \lambda^{2}\right)\right)\right)\right)\right)+2 \alpha \lambda\left(y_{-2}(\alpha)(\beta(-36\right.$ $\left.+3 \ell^{3} \alpha^{2} \lambda^{2}+2\left(-12+\alpha^{2}\right) \lambda^{2} \ell^{4}\left(36-2\left(-12+\alpha^{2}\right) \lambda^{2}\right)\right)+\alpha \lambda$ $\left(2\left(9+\left(6+\alpha^{2}\right) \lambda^{2}\right)-2 \ell^{4}\left(9+\left(6+\alpha^{2}\right) \lambda^{2}\right)+3 \ell^{3}(27+(18+\right.$ $\left.\left.\left.\left.\alpha^{2}\right) \lambda^{2}\right)\right)\right)+\alpha^{2}\left(6 y_{-1}(\alpha \ell) \ell^{2}(2 \beta-\alpha \lambda)\left(3+2 \lambda^{2}\right)+y_{-1}(\alpha)((\right.$ $\left.-2-3 \ell^{3}+2 \ell^{4}\right) \alpha \lambda\left(3+2 \lambda^{2}\right)+\beta\left(12-2\left(-4+\alpha^{2}\right) \lambda^{2}+2 \ell^{4}(\right.$ $\left.\left.\left.\left.\left.-6+\left(-4+\alpha^{2}\right) \lambda^{2}\right)-3 \ell^{3}\left(12+\left(8+\alpha^{2}\right) \lambda^{2}\right)\right)\right)\right)\right)+2 m^{6}\left(y_{-2}(\alpha\right.$ )$\left(-2 \alpha^{3} \beta \lambda^{3}-2 \alpha^{4} \lambda^{4}-54\left(-1+\lambda^{2}\right)+3 \alpha \beta \lambda\left(-9+8 \lambda^{2}\right)+\alpha^{2}\right.$ $\left(-9 \lambda^{2}-12 \lambda^{4}\right)-3 \ell^{3} \alpha^{2} \lambda^{2}\left(\alpha \beta \lambda+\alpha^{2} \lambda^{2}+18\left(-1+\lambda^{2}\right)\right)+\ell^{4}$ $\left(2 \alpha^{3} \beta \lambda^{3}+2 \alpha^{4} \lambda^{4}+54\left(-1+\lambda^{2}\right)+3 \alpha^{2} \lambda^{2}\left(-3+4 \lambda^{2}\right)-3 \alpha \beta\right.$ $\left.\left.\lambda\left(-9+8 \lambda^{2}\right)\right)\right)+\alpha^{2}\left(3 y_{-1}(\alpha \ell) \ell^{2}\left(\alpha \beta \lambda\left(9-8 \lambda^{2}\right)+18\left(-1+\lambda^{2}\right.\right.\right.$ )$\left.+\alpha^{2} \lambda^{2}\left(-3+4 \lambda^{2}\right)\right)+y_{-1}(\alpha)\left(2 \alpha^{3} \beta \lambda^{3}+\alpha \beta \lambda\left(-9+8 \lambda^{2}\right)+\right.$ $18\left(-1+\lambda^{2}\right)+\alpha^{2}\left(-6 \lambda^{2}+4 \lambda^{4}\right)+3 \ell^{3}\left(\alpha^{3} \beta \lambda^{3}-18\left(-1+\lambda^{2}\right)\right.$ $\left.+\alpha^{2} \lambda^{2}\left(-3+2 \lambda^{2}\right)+\alpha \beta \lambda\left(-9+8 \lambda^{2}\right)\right)+\ell^{4}\left(-2 \alpha^{3} \beta \lambda^{3}-18(-\right.$ $\left.\left.\left.\left.\left.1+\lambda^{2}\right) \quad \alpha \beta \lambda\left(-9+8 \lambda^{2}\right)+\alpha^{2}\left(6 \lambda^{2}-4 \lambda^{4}\right)\right)\right)\right)\right)+3 m\left(y_{-2}(\alpha)\right.$ $\left(-3 \ell^{3} \alpha^{2} \lambda^{2}\left(2+\alpha \beta \lambda+18 \lambda^{2}+\alpha^{2} \lambda^{2}\right)-2\left(\alpha^{3} \beta \lambda^{3}+\alpha^{4} \lambda^{4}+6\right.\right.$ $\left.\alpha \beta \lambda\left(1-2 \lambda^{2}\right)+6\left(3+2 \lambda^{2}\right) \alpha^{2}\left(8 \lambda^{2}+6 \lambda^{4}\right)\right)+2 \ell^{4}\left(\alpha^{3} \beta \lambda^{3}+\right.$ $\left.\left.\alpha^{4} \lambda^{4}+6 \alpha \beta \lambda\left(1-2 \lambda^{2}\right)+6\left(3+2 \lambda^{2}\right) \alpha^{2}\left(8 \lambda^{2}+6 \lambda^{4}\right)\right)\right)+\alpha^{2}$ $\left(6 y_{-1}(\alpha) \ell^{2}\left(6+4 \lambda^{2}\right)+\alpha \beta\left(2 \lambda-4 \lambda^{3}\right)+\alpha^{2}\left(\lambda^{2}+2 \lambda^{4}\right)\right)+$ $y_{-1}(\alpha)\left(2\left(6+4 \lambda^{2}+\alpha^{3} \beta \lambda^{3}+\alpha \beta\left(2 \lambda-4 \lambda^{3}\right)+2 \alpha^{2}\left(\lambda^{2}+\lambda^{4}\right.\right.\right.$ )$)-2 \ell^{4}\left(6+4 \lambda^{2}+\alpha^{3} \beta \lambda^{3}+\alpha \beta\left(2 \lambda-4 \lambda^{3}\right)+2 \alpha^{2}\left(\lambda^{2}+\lambda^{4}\right)\right.$ )$+3 \ell^{3}\left(\alpha^{3} \beta \lambda^{3}-4\left(3+2 \lambda^{2}\right)+\alpha \beta\left(-4 \lambda+8 \lambda^{3}\right)+2 \alpha^{2}\left(\lambda^{2}+\right.\right.$ 
$\left.\left.\left.\left.\left.\left.\lambda^{4}\right)\right)\right)\right)\right)\right)+y_{-2}(\alpha \ell)\left(-3 m^{5} \alpha \lambda\left(y_{2}(\alpha)\left(\beta\left(24+3 \ell^{3} \alpha^{2} \lambda^{2}+2(-\right.\right.\right.\right.$ $\left.12+\alpha^{2}\right) \lambda^{2}+\alpha^{3} \beta \lambda^{3}-4\left(3+2 \lambda^{2}\right)+\alpha \beta\left(-4 \lambda+8 \lambda^{3}\right)-2 \ell^{4}$ $\left.\left(12+\left(-12+\alpha^{2}\right) \lambda^{2}\right)\right)+\alpha \lambda\left(2\left(-6+\left(6+\alpha^{2}\right) \lambda^{2}\right)-2 \ell^{4}(-6\right.$ $\left.\left.\left.+\left(6+\alpha^{2}\right) \lambda^{2}\right)+3 \ell^{3}\left(-18+\left(18+\alpha^{2}\right) \lambda^{2}\right)\right)\right)+\alpha^{2}\left(12 y_{1}(\alpha \ell)\right.$ $\ell^{2}(2 \beta-\alpha \lambda)\left(-1+\lambda^{2}\right)+y_{1}(\alpha)\left(2\left(-2-3 \ell^{3}+2 \ell^{4}\right) \alpha \lambda(-1+\right.$ $\left.\lambda^{2}\right)+\beta\left(-8-2\left(-4+\alpha^{2}\right) \lambda^{2}+2 \ell^{4}\left(4+\left(-4+\alpha^{2}\right) \lambda^{2}\right)-3 \ell^{3}\right.$ $\left.\left.\left.\left.\left(-8+\left(8+\alpha^{2}\right) \lambda^{2}\right)\right)\right)\right)\right)+2 \alpha \lambda\left(y_{2}(\alpha)\left(\beta\left(-36+3 \ell^{3} \alpha^{2} \lambda^{2}-2\right.\right.\right.$ $\left.\left(-12+\alpha^{2}\right) \lambda^{2}+2 \ell^{4}\left(-18+\left(-12+\alpha^{2}\right) \lambda^{2}\right)\right)+\alpha \lambda(-2(9+($ $\left.\left.6+\alpha^{2}\right) \lambda^{2}\right)+2 \ell^{4}\left(9+\left(6+\alpha^{2}\right) \lambda^{2}\right)-3 \ell^{3}\left(27+\left(18+\alpha^{2}\right) \lambda^{2}\right)$ )$)+\alpha^{2}\left(-6 y_{1}(\alpha \ell) \ell^{2}(2 \beta-\alpha \lambda)\left(3+2 \lambda^{2}\right)+y_{1}(\alpha)(-(-2-3\right.$ $\left.\ell^{3}+2 \ell^{4}\right) \alpha \lambda\left(3+2 \lambda^{2}\right)+\beta\left(\ell^{4}\left(12-2\left(-4+\alpha^{2}\right) \lambda^{2}\right)+2(-6\right.$ $\left.\left.\left.\left.\left.+\left(-4+\alpha^{2}\right) \lambda^{2}\right)+3 \ell^{3}\left(12+\left(8+\alpha^{2}\right) \lambda^{2}\right)\right)\right)\right)\right)-2 m^{6}\left(y_{2}(\alpha)(-2\right.$ $\alpha^{3} \beta \lambda^{3}-2 \alpha^{4} \lambda^{4}-54\left(-1+\lambda^{2}\right)+3 \alpha \beta \lambda\left(-9+8 \lambda^{2}\right)+\alpha^{2}(-9$ $\left.\lambda^{2}-12 \lambda^{4}\right)-3 \ell^{3} \alpha^{2} \lambda^{2}\left(\alpha \beta \lambda+\alpha^{2} \lambda^{2}+18\left(-1+\lambda^{2}\right)\right)+\ell^{4}\left(2 \alpha^{3}\right.$ $\beta \lambda^{3}+2 \alpha^{4} \lambda^{4}+54\left(-1+\lambda^{2}\right)+3 \alpha^{2} \lambda^{2}\left(-3+4 \lambda^{2}\right)-3 \alpha \beta \lambda(-9$ $\left.\left.\left.+8 \lambda^{2}\right)\right)\right)+\alpha^{2}\left(3 y_{1}(\alpha \ell) \ell^{2}\left(\alpha \beta \lambda\left(9-8 \lambda^{2}\right)+18\left(-1+\lambda^{2}\right)+\alpha^{2}\right.\right.$ $\left.\lambda^{2}\left(-3+4 \lambda^{2}\right)\right)+y_{1}(\alpha)\left(2 \alpha^{3} \beta \lambda^{3}+\alpha \beta \lambda\left(9-8 \lambda^{2}\right)+18(-1+\right.$ $\left.\lambda^{2}\right)+\alpha^{2}\left(-6 \lambda^{2}+4 \lambda^{4}\right)+3 \ell^{3}\left(\alpha^{3} \beta \lambda^{3}-18\left(-1+\lambda^{2}\right)+\alpha^{2} \lambda^{2}\right.$ $\left.\left(-3+2 \lambda^{2}\right)+\alpha \beta \lambda\left(-9+8 \lambda^{2}\right)\right)+\ell^{4}\left(-2 \alpha^{3} \beta \lambda^{3}-18\left(-1+\lambda^{2}\right)\right.$ $\left.\left.\left.\left.\alpha \beta \lambda\left(-9+8 \lambda^{2}\right)+\alpha^{2}\left(6 \lambda^{2}-4 \lambda^{4}\right)\right)\right)\right)\right)-3 m\left(y_{2}(\alpha)\left(-3 \ell^{3} \alpha^{2} \lambda^{2}\right.\right.$ $\left(2+\alpha \beta \lambda+18 \lambda^{2}+\alpha^{2} \lambda^{2}\right)-2\left(\alpha^{3} \beta \lambda^{3}+\alpha^{4} \lambda^{4}+6 \alpha \beta \lambda\left(1-2 \lambda^{2}\right)\right.$ $\beta \lambda^{3}-2 \alpha^{4} \lambda^{4}-54\left(-1+\lambda^{2}\right)+3 \alpha \beta \lambda\left(-9+8 \lambda^{2}\right)+\alpha^{2}\left(-9 \lambda^{2}-\right.$ $\left.12 \lambda^{4}\right)-3 \ell^{3} \alpha^{2} \lambda^{2}\left(\alpha \beta \lambda+\alpha^{2} \lambda^{2}+18\left(-1+\lambda^{2}\right)\right)+\ell^{4}\left(2 \alpha^{3} \beta \lambda^{3}+\right.$ $\left.\left.2 \alpha^{4} \lambda^{4}+54\left(-1+\lambda^{2}\right)+3 \alpha^{2} \lambda^{2}\left(-3+4 \lambda^{2}\right)-3 \alpha \beta \lambda\left(-9+8 \lambda^{2}\right)\right)\right)$ $+\alpha^{2}\left(3 y_{1}(\alpha \ell) \ell^{2}\left(\alpha \beta \lambda\left(9-8 \lambda^{2}\right)+18\left(-1+\lambda^{2}\right)+\alpha^{2} \lambda^{2}(-3+4\right.\right.$ $\left.\left.\lambda^{2}\right)\right)+y_{1}(\alpha)\left(2 \alpha^{3} \beta \lambda^{3}+\alpha \beta \lambda\left(9-8 \lambda^{2}\right)+18\left(-1+\lambda^{2}\right)+\alpha^{2}(-\right.$ $\left.6 \lambda^{2}+4 \lambda^{4}\right)+3 \ell^{3}\left(\alpha^{3} \beta \lambda^{3}-18\left(-1+\lambda^{2}\right)+\alpha^{2} \lambda^{2}\left(-3+2 \lambda^{2}\right)+\right.$ $\left.\alpha \beta \lambda\left(-9+8 \lambda^{2}\right)\right)+\ell^{4}\left(-2 \alpha^{3} \beta \lambda^{3}-18\left(-1+\lambda^{2}\right) \alpha \beta \lambda\left(-9+8 \lambda^{2}\right)\right.$ $\left.\left.\left.\left.+\alpha^{2}\left(6 \lambda^{2}-4 \lambda^{4}\right)\right)\right)\right)\right)-3 m\left(y_{2}(\alpha)\left(-3 \ell^{3} \alpha^{2} \lambda^{2}\left(2+\alpha \beta \lambda+18 \lambda^{2}\right.\right.\right.$ $\left.+\alpha^{2} \lambda^{2}\right)-2\left(\alpha^{3} \beta \lambda^{3}+\alpha^{4} \lambda^{4}+6 \alpha \beta \lambda\left(1-2 \lambda^{2}\right)+6\left(3+2 \lambda^{2}\right)+\alpha^{2}\right.$ $\left.\left(8 \lambda^{2}+6 \lambda^{4}\right)\right)+2 \ell^{4}\left(\alpha^{3} \beta \lambda^{3}+\alpha^{4} \lambda^{4}+6 \alpha \beta \lambda\left(1-2 \lambda^{2}\right)+6(3+2\right.$ $\left.\left.\left.\lambda^{2}\right)+\alpha^{2}\left(8 \lambda^{2}+6 \lambda^{4}\right)\right)\right)+\alpha^{2}\left(6 y_{1}(\alpha \ell) \ell^{2}\left(6+4 \lambda^{2}\right)+\alpha \beta(2 \lambda-\right.$ $\left.\left.4 \lambda^{3}\right)+\alpha^{2}\left(\lambda^{2}+2 \lambda^{4}\right)\right)+y_{1}(\alpha)\left(2\left(6+4 \lambda^{2}+\alpha^{3} \beta \lambda^{3}+\alpha \beta(2 \lambda-\right.\right.$ $\left.\left.4 \lambda^{3}\right)+2 \alpha^{2}\left(\lambda^{2}+\lambda^{4}\right)\right)-2 \ell^{4}\left(6+4 \lambda^{2}+\alpha^{3} \beta \lambda^{3}+\alpha \beta(2 \lambda-\right.$ $\left.\left.4 \lambda^{3}\right)+2 \alpha^{2}\left(\lambda^{2}+\lambda^{4}\right)\right)+3 \ell^{3}\left(\alpha^{3} \beta \lambda^{3}-4\left(3+2 \lambda^{2}\right)+\alpha \beta\left(-4 \lambda+8 \lambda^{3}\right)\right.$ $\left.\left.\left.\left.\left.\left.+2 \alpha^{2}\left(\lambda^{2}+\lambda^{4}\right)\right)\right)\right)\right)\right)\right]$

where, $\ell=a / b$ and $m=c / b$, are dimensionless parameters with $y_{1}(\alpha)=\sinh \alpha$ and $y_{-1}(\alpha)=\cosh \alpha$

\section{Appendix B}

$C_{2}^{*}=\lambda\left[y_{2}(\alpha \ell) \alpha\left\{y_{-2}(\alpha)\left(24 \beta\left(-1+\ell^{4}\right)+\left(-24\left(-1+\ell^{4}\right)+(\right.\right.\right.\right.$ $\left.\left.-2+\ell^{3}(-3+2 \ell)\right) \alpha^{2}\right) \beta \lambda^{2}+\left(-2+\ell^{3}(-3+2 \ell)\right) \alpha^{3} \lambda^{3}+6(-2$ $\left.\left.+\ell^{3}(-9+2 \ell)\right) \alpha \lambda\left(-1+\lambda^{2}\right)\right)+\alpha^{2}\left(-12 y_{-1}(\alpha \ell) \ell^{2}(-2 \beta+\alpha \lambda)\right.$ $\left(-1+\lambda^{2}\right)+y_{-1}(\alpha)\left(-8\left(-1+\ell^{3}(3+\ell)\right) \beta-\left(8-2 \alpha^{2}+\ell^{3}(-\right.\right.$ $\left.\left.8(3+\ell)+(-3+2 \ell) \alpha^{2}\right)\right) \beta \lambda^{2}-2\left(-2+\ell^{3}(-3+2 \ell)\right) \alpha(-1+$ $\left.\left.\left.\left.\lambda^{2}\right) \lambda\right)\right)\right\}+y_{-2}(\alpha \ell) \alpha\left\{y_{2}(\alpha)\left(-24 \beta\left(-1+\ell^{4}\right)-\left(-24\left(-1+\ell^{4}\right.\right.\right.\right.$ )$\left.+\left(-2+\ell^{3}(-3+2 \ell)\right) \alpha^{2}\right) \beta \lambda^{2}-\left(-2+\ell^{3}(-3+2 \ell)\right) \alpha^{3} \lambda^{3}-$ $\left.6\left(-2+\ell^{3}(-9+2 \ell)\right) \alpha \lambda\left(-1+\lambda^{2}\right)\right)+\alpha^{2}\left(-12 y_{1}(\alpha \ell) \ell^{2}(-2 \beta\right.$ $+\alpha \lambda)\left(-1+\lambda^{2}\right)+y_{1}(\alpha)\left(8\left(-1+\ell^{3}(3+\ell)\right) \beta+\left(8-2 \alpha^{2}+\ell^{3}\right.\right.$ $\left.\left(-8(3+\ell)+(-3+2 \ell) \alpha^{2}\right)\right) \beta \lambda^{2}+2\left(-2+\ell^{3}(-3+2 \ell)\right) \alpha(-1$ $\left.\left.\left.\left.+\lambda^{2}\right) \lambda\right)\right)\right\}+\ell \alpha^{3}\left\{-4\left(y_{-2}(\alpha) y_{1}(\alpha)-y_{-1}(\alpha) y_{2}(\alpha)\right) \ell\left(2+\ell^{4}\right)\right.$ $(2 \beta-\alpha \lambda)\left(-1+\lambda^{2}\right)+y_{-1}(\alpha \ell)\left(y_{1}(\alpha) \alpha^{2}\left(-8\left(-1+\ell^{3}\right) \beta+(\right.\right.$ $\left.\left.2\left(-4+\alpha^{2}\right)+\ell^{3}\left(8+\alpha^{2}\right)\right) \beta \lambda^{2}+2\left(2+\ell^{3}\right) \alpha \lambda\left(-1+\lambda^{2}\right)\right)+y_{2}$ $(\alpha)\left(-\left(2+\ell^{3}\right) \alpha^{3} \lambda^{3}-6\left(2+3 \ell^{3}\right) \alpha \lambda\left(-1+\lambda^{2}\right)+\beta(-24-(-\right.$ $\left.\left.\left.\left.24+\left(2+\ell^{3}\right) \alpha^{2}\right) \lambda^{2}\right)\right)\right\} y_{1}(\alpha \ell)\left(y_{-1}(\alpha) \alpha^{2}\left(8\left(-1+\ell^{3}\right) \beta-(2(\right.\right.$ $\left.\left.\left.-4+\alpha^{2}\right)+\ell^{3}\left(8+\alpha^{2}\right)\right) \beta \lambda^{2}-2\left(2+\ell^{3}\right) \alpha \lambda\left(-1+\lambda^{2}\right)\right)+y_{-2}($ $\alpha)\left(\left(2+\ell^{3}\right) \alpha^{3} \lambda^{3}+6\left(2+3 \ell^{3}\right) \alpha \lambda\left(-1+\lambda^{2}\right)+\beta(24+(-24+\right.$ $\left.\left.\left.\left.\left.\left.\left(2+\ell^{3}\right) \alpha^{2}\right) \lambda^{2}\right)\right)\right)\right\}\right] / \Delta_{1}$,

$\Delta_{1}=2\left[18\left\{3 y_{-2}(\alpha \ell) y_{2}(\alpha)\left(-1+\ell^{4}\right)-\left(\ell\left(3 y_{1}(\alpha \ell) y_{-2}(\alpha)-3\right.\right.\right.\right.$ $\left.y_{-1}(\alpha \ell) y_{2}(\alpha)-\left(y_{1}(\alpha) y_{-2}(\alpha)-y_{2}(\alpha) y_{-1}(\alpha)\right) \ell\left(2+\ell^{4}\right)\right)+$ $\left.y_{-2}(\alpha \ell)\left(-3 y_{1}(\alpha \ell) \ell^{2}+y_{1}(\alpha)\left(-1+\ell^{3}(3+\ell)\right)\right)\right) \alpha^{2}+\left(-y_{1}(\alpha\right.$

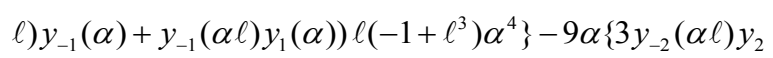
$(\alpha)\left(-1+\ell^{4}\right)-\left(\ell\left(3 y_{1}(\alpha \ell) y_{-2}(\alpha)-3 y_{-1}(\alpha \ell) y_{2}(\alpha)-\left(y_{1}(\alpha)\right.\right.\right.$ $\left.\left.y_{-2}(\alpha)-y_{2}(\alpha) y_{-1}(\alpha)\right) \ell\left(2+\ell^{4}\right)\right)+y_{-2}(\alpha \ell)\left(-3 y_{1}(\alpha \ell) \ell^{2}+\right.$ $\left.\left.y_{1}(\alpha)\left(-1+\ell^{3}(3+\ell)\right)\right)\right) \alpha^{2}+\left(-y_{1}(\alpha \ell) y_{-1}(\alpha)+y_{-1}(\alpha \ell) y_{1}(\alpha)\right.$ $\left.\ell\left(-1+\ell^{3}\right) \alpha^{4}\right\} \beta \lambda 3\left\{y_{-2}(\alpha \ell)\left(-18 y_{2}(\alpha)\left(-1+\ell^{4}\right)+3\left(-6 y_{1}(\alpha\right.\right.\right.$ $\left.\ell) \ell^{2}+y_{2}(\alpha)\left(-1+(-6+\ell) \ell^{3}\right)+2 y_{1}(\alpha)\left(-1+\ell^{3}(3+\ell)\right)\right) \alpha^{2}+($ $\left.\left.3 y_{1}(\alpha \ell) \ell^{2}+y_{1}(\alpha)\left(2+(3-2 \ell) \ell^{3}\right)\right) \alpha^{4}\right)+\ell \alpha^{2}\left(\left(y_{1}(\alpha) y_{-2}(\alpha)-\right.\right.$ $\left.y_{2}(\alpha) y_{-1}(\alpha)\right) \ell\left(2+\ell^{4}\right)\left(-6+\alpha^{2}\right)+y_{1}(\alpha \ell)\left(18 y_{-2}(\alpha)-3\left(2 y_{-1}\right.\right.$ $\left.\left.(\alpha)+y_{-2}(\alpha)-2\left(y_{-1}(\alpha)-y_{-2}(\alpha)\right) \ell^{3}\right) \alpha^{2}+y_{-1}(\alpha)\left(2+\ell^{3}\right) \alpha^{4}\right)$ $+y_{-1}(\alpha \ell)\left(-18 y_{2}(\alpha)+3\left(2 y_{1}(\alpha)+y_{2}(\alpha)-2\left(y_{1}(\alpha)-y_{2}(\alpha)\right) \ell^{3}\right.\right.$ )$\left.\left.\left.\alpha^{2}-y_{1}(\alpha)\left(2+\ell^{3}\right) \alpha^{4}\right)\right)\right\} \lambda^{2}+\alpha\left(24 y_{-2}(\alpha \ell) y_{2}(\alpha)\left(-1+\ell^{4}\right)-\right.$ $\left(-8 \ell\left(-3 y_{1}(\alpha \ell) y_{-2}(\alpha)+3 y_{-1}(\alpha \ell) y_{2}(\alpha)+\left(y_{1}(\alpha) y_{-2}(\alpha)-y_{2}\right.\right.\right.$ $\left.\left.(\alpha) y_{-1}(\alpha)\right) \ell\left(2+\ell^{4}\right)\right)+y_{-2}(\alpha \ell)\left(-24 y_{1}(\alpha \ell) \ell^{2}+8 y_{1}(\alpha)(-1+\right.$ $\left.(3+\ell) \ell^{3}\right)$

$\left.\left.\left.+y_{2}(\alpha)\left(-2+\ell^{3}(-3+2 \ell)\right)\right)\right) \alpha^{2}\right)+\left(2 y_{1}(\alpha)\left(4 y_{-1}(\alpha)+y_{-2}(\alpha)\right)\right.$ $\ell-2 y_{-1}(\alpha \ell)\left(4 y_{1}(\alpha)+y_{2}(\alpha)\right) \ell+\left(-8 y_{1}(\alpha \ell) y_{-1}(\alpha)+y_{1}(\alpha \ell)\right.$ $\left.y_{-2}(\alpha)+8 y_{-1}(\alpha \ell) y_{1}(\alpha)-y_{-1}(\alpha \ell) y_{2}(\alpha)\right) \ell^{4}+y_{-2}(\alpha \ell) y_{1}(\alpha)$ $\left.\left(-2+\ell^{3}(-3+2 \ell)\right)\right) \alpha^{4}+\left(-y_{1}(\alpha \ell) y_{-1}(\alpha)+y_{-1}(\alpha \ell) \ell\left(2+\ell^{3}\right)\right.$ $\left.\alpha^{6}\right) \beta \lambda^{3}+\left(-6 y_{-2}(\alpha \ell) y_{2}(\alpha)\left(-2+\ell^{3}(-9+2 \ell)\right) \alpha^{2}+(-2 \ell(-3\right.$ $y_{1}(\alpha \ell) y_{-2}(\alpha)\left(2+3 \ell^{3}\right)+3 y_{-1}(\alpha \ell) y_{2}(\alpha)\left(2+3 \ell^{3}\right)+2\left(y_{1}(\alpha)\right.$ $\left.\left.y_{-2}(\alpha)-y_{2}(\alpha) y_{-1}(\alpha)\right) \ell\left(2+\ell^{4}\right)\right)+y_{-2}(\alpha \ell)\left(-12 y_{1}(\alpha \ell) \ell^{2}+\right.$ $\left.\left.y_{2}(\alpha)\left(2+\ell^{3}(3-2 \ell)\right)+y_{1}(\alpha)\left(-4-6 \ell^{3}+4 \ell^{4}\right)\right)\right) \alpha^{4}+\left(-2 y_{1}(\alpha\right.$

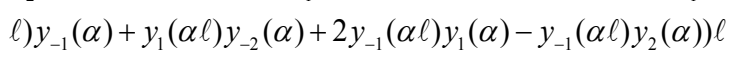
$\left.\left(2+\ell^{3}\right) \alpha^{6}\right) \lambda^{4}+y_{2}(\alpha \ell)\left(y_{-2}(\alpha)\left(-54\left(-1+\ell^{4}\right)+27\left(-1+\ell^{4}\right) \alpha\right.\right.$ $\beta \lambda-9\left(6-6 \ell^{4}+\left(-1+(-6+\ell) \ell^{3}\right) \alpha^{2}\right) \lambda^{2}+\alpha\left(-24\left(-1+\ell^{4}\right)+\right.$ $\left.\left(-2+\ell^{3}(-3+2 \ell)\right) \alpha^{2}\right) \beta \lambda^{3}+\alpha^{2}\left(-2\left(6+\alpha^{2}\right)+2 \ell^{4}\left(6+\alpha^{2}\right)-3\right.$ 


$$
\begin{aligned}
& \left.\left.\ell^{3}\left(18+\alpha^{2}\right)\right) \lambda^{4}\right)+\alpha^{2}\left(y _ { - 1 } ( \alpha ) \left(18\left(-1+\ell^{3}(3+\ell)\right)-9\left(-1+\ell^{3}(3\right.\right.\right. \\
& +\ell)) \alpha \beta \lambda+3\left(3\left(6-2 \alpha^{2}+\ell^{3}\left(-6(3+\ell)+(-3+2 \ell) \alpha^{2}\right)\right) \lambda^{2}-\alpha\right. \\
& \left(8-2 \alpha^{2}+\ell^{3}\left(-8(3+\ell)+(-3+2 \ell) \alpha^{2}\right)\right) \beta \lambda^{3}-2\left(-2+\ell^{3}(-3\right. \\
& \left.+2 \ell)) \alpha^{2} \lambda^{4}\right)+3 y_{-1}(\alpha \ell) \ell^{2}\left(-18+\lambda\left(18 \lambda+\alpha\left(9 \beta-3 \alpha \lambda-8 \beta \lambda^{2}\right.\right.\right. \\
& \left.\left.\left.\left.\left.\left.+4 \alpha \lambda^{3}\right)\right)\right)\right)\right)\right] \\
& C_{3}^{*}=\alpha \beta \lambda\left[\left\{\left(-1+m^{5}\right)(36 \beta-18 \lambda \alpha)+\left(2+3 m^{5}\right)\left(-12 \beta \lambda^{2}\right.\right.\right. \\
& \left.\left.+6 \alpha \lambda^{3}+\alpha^{2} \beta \lambda^{2}+\alpha^{3} \lambda^{3}\right)\right\} y_{2}(\alpha)+\left\{( 2 + 3 m ^ { 5 } ) \left(4 \beta \lambda^{2} \alpha^{2}-2 \alpha^{3} \lambda^{3}\right.\right. \\
& \left.\left.\left.-\alpha^{4} \beta \lambda^{2}\right)+\left(-1+m^{5}\right)\left(-12 \beta \alpha^{2}+6 \alpha^{3} \lambda\right)\right\} y_{1}(\alpha)\right] / \Delta_{2}, \\
& \Delta_{2}=\left[-54 m\left(-1+m^{5}\right)+9\left(4+2 m-4 m^{5}+3 m^{6}\right) \beta \lambda^{2} \alpha+18 m(2\right. \\
& \left.+3 m^{5}\right) \lambda^{2}+(-1+m)^{3}(1+m)\left(2+m+2 m^{2}\right)\left(-12 \beta \lambda^{3} \alpha+\beta \lambda^{3} \alpha^{3}\right. \\
& \left.+6 \lambda^{4} \alpha^{2}+\lambda^{4} \alpha^{4}\right)+\lambda^{2} \alpha^{2}( \\
& \left.\left.-18+24 m+18 m^{5}-9 m^{6}\right)\right] y_{2}(\alpha)+\left[(-1+m)^{3}(1+\right. \\
& m)\left(2+m+2 m^{2}\right)\left(4 \beta \lambda^{3} \alpha^{3}-\beta \lambda^{3} \alpha^{5}-2 \lambda^{4} \alpha^{4}\right)+18 \\
& m\left(-1+m^{5}\right) \alpha^{2}-3\left(4+2 m-4 m^{5}+3 m^{6}\right) \beta \lambda \alpha^{3}+6 \\
& (-1+m)^{2}\left(1+m+m^{2}+m^{3}+m^{4}\right) \lambda^{2} \alpha^{4}-12 m \lambda^{2} \alpha^{2} \\
& \left.-18 m^{6} \lambda^{2} \alpha^{2}\right] y_{1}(\alpha) .
\end{aligned}
$$

\section{REFERENCES}

[1] Brinkman H. C., 1947, A calculation of viscous force exerted by a flowing fluid on a dense swarm of particles, J. Appl. Sci. Res. A1: $27-34$

[2] Uchida, S., 1954, Viscous flow in multiparticle systems: slow viscous flow through a mass of particles, Int. Sci. Technol. Univ. Tokyo (in Japanese) 3; 97- Abstract: Ind. Engng. Chem. 46, 1194-1195 (translated by T. Motai)

[3] Brenner H., 1957, Eng. Sc. D. thesis, New York University

[4] Happel J., 1958, Viscous flow in multiparticle system: Slow motion of fluids relative to beds of spherical particles, J A I Ch E 4(2): 197-201

[5] Happel J., 1959, Viscous flow relative to arrays of cylinders, A.I.Ch.E.5 (2):174-177

[6] Deo, S., Yadav, P.K. and Tiwari, A., 2010, Slow viscous flow through a membrane built up from porous cylindrical particles with an impermeable core, Appl. Math. Mod., 34, $1329-1343$

[7] Jones I. P., 1973, Low Reynolds number flow past a porous spherical shell, Proc. Camb. Philos. Soc. 73: 231-238

[8] Qin Yu, and Kaloni P. N., 1988, A Cartesian tensor solution of the Brinkman equation, J. Engng Math 22: 177-188

[9] Davis R. H. and Stone H. A., 1993, Flow through beds of porous particles. Chem Engng Sci 48 (23) 3993-4005

[10] Bhatt B. S., 1993, Slow extensional flow Past a sphere, Modelling Measurement Cont B ASME 51(1), 167-177

[11] Padmavati B. S., Amaranath T., Palaniappan D., 1994, Stokes flow past a permeable sphere, Non-axisymmetric case ZAMM 74(7): 290-292

[12] Givler R. C. and Altobelli S. A., 1994, A determination of the effective viscosity for the Brinkman Forchheimer flow model, J. Fluid Mech. 258, 355-370

[13] Dassios G., 1994, Hadjinicolaou M. and Payatakes A. C.: Generalized eigenfunctions and complete semiseparable solutions for Stokes flow in spheroidal co-ordinates, Q. Appl. Math. 52, 157-191

[14] Dassios G., Hadjinicolaou M., Coutelieris F. A. and Payatakes A. C., 1995 Stokes flow in spheroidal particle-in-cell models with Happel and Kuwabara boundary conditions, Int. J. Engng. Sci. 33(10), 1465-1490

[15] Ochoa-Tapia J. A., Whitaker S., 1995(a), Momentum transfer at the boundary between a porous medium and a heterogeneous fluid-I, Theoretical development, Int. J. Heat Mass Transfer 38:2635-2646

[16] Ochoa-Tapia J. A., Whitaker S., 1995 (b), Momentum transfer at the boundary between a porous medium and a heterogeneous fluid-II, comparison with experiment. Int J Heat Mass Transfer38: 2647-2655

[17] Kuznetsov A. V., 1996, Analytical investigation of the fluid flow in the interface region between a porous media and a clear fluid in channels partially filled with porous medium, Appl. Sci Res. 56: 53-67

[18] Kuznetsov A. V., 1998, Analytical investigation of Couette flow in a composite channel partially filled with a porous media and partially with a clear fluid, Int. J. Heat Mass transfer 41, 2556-2560

[19] Zlatanovski, T., 1999, Axisymmetric creeping flow past a porous prolate spheroidal particle using the Brinkman model, Quart. J. Mech. Appl. Math. 52(1), 111-126

[20] Raja-Sekhar G. P., Amaranath T., 2000, Stokes flow inside a porous spherical shell, Ziet Angew Math Phys 51 No.-3: 481- 490

[21] Bhatt B. S. and Shirley A., 2001, Slow extensional bounded flows, Differential equations and control processes N 1: 1-9

[22] Datta, S. and Deo S., 2002, Stokes flow with slip and $\mathrm{Ku}-$ wabara boundary conditions, Proc. Ind. Acad. Sci. (Math. Sci.), 112 (3), 463-475

[23] Bhattacharyya A., Raja Sekhar G. P., 2004, Viscous flow past a porous sphere with an impermeable core:effect of stress jump condition, Chem Eng Sci 59: 4481-4492

[24] Bhattacharyya A., Raja Sekhar G. P., 2005, Stokes flow inside a porous spherical shell: Stress jump boundary condition, ZAMP 56: 475-496

[25] Srivastava A. C., Srivastava N., 2005, Flow past a porous sphere at small Reynolds numbers, ZAMP 56: 821-835

[26] Deo, S. and Yadav, P. K., 2008, Creeping flow past a swarm of porous deformed oblate spheroidal particles with kuwabara boundary condition, Bull. Cal. Math. Soc., 100, (6) 617-630

[27] Chandesris M., Jamet D., 2006, Boundary conditions at a planar fluid-porous interface for a Poiseuille flow, Int J Heat Mass Transfer 49: 2137-2150

[28] Valdes-Parada F. J., Goyeau B., Ochoa-Tapia J. A., 2006, Diffusive mass transfer between a microporous medium and an homogeneous fluid: jump boundary condition, Chem 
Engng Sci 61: 1692-1704

[29] Chandesris M., Jamet D., 2007, Boundary conditions at a fluid-porous interface: An a priori estimation of the stress jump coefficients, Int J Heat Mass Transfer50: 3422-3436.

[30] Valdes-Parada F. J., Goyeau B., Ochoa-Tapia J A., 2007, Jump momentum boundary condition at a fluid-porous dividing surface: derivation of the closure problem.between a microporous medium and an homogeneous fluid: jump boundary condition, Chem Engng Sci 61: 1692-1704 (2006) Chem Engng Sci 62: 4025-4039

[31] Happel J., Brenner H., 1983, Low Reynolds Number Hydrodynamics Martinus Nijoff,, The Hague,Netherlands

[32] Abramowitz M., Stegun I. A., 1970, Handbook of Mathematical Functions. Dover Publications, New York 